\title{
El Proceso de Terciarización en los Municipios Metropolitanos del Estado de México
}

\author{
Guadalupe Hoyos Castillo \\ Centro de Investigación Y Estudios Avanzados de la Población \\ UAEM
}

\section{Introducción}

La concentración espacial del crecimiento del sector terciario es un fenómeno que ha venido acrecentando su papel en la vida económica nacional, al nivel regional, urbana y en las zonas metropolitanas. La estructura de la oferta ocupacional global de México se ha modificado al mismo tiempo que incrementado la participación del sector terciario. En 1950, 25 por ciento de la PEA nacional se ubicaba en el terciario para alcanzar en 1990,46 por ciento del total. En el Estado de México la transferencia poblacional es más importante aún, el sector significaba sólo 12 por ciento en 1950, para luego alcanzar 53 por ciento en 1990. La aportación del sector al producto nacional durante los últimos veinte años significa poco más de la mitad.

La concentración sectorial de la economía mexicana principalmente exhibe que el empleo global se basa en actividades generadas en el terciario, que la población trabajadora "empuja" en éste porque en la manufactura y actividades agropecuarias hay bajas posibilidades de captación. Presentándose altas tasas de crecimiento en el comercio y los servicios tradicionales. Aunque existen también servicios con mejores rendimientos, generalmente eslabonados con ramas industriales prósperas pero con participación en empleo baja. La proporción de participación del sector en la generación del producto nacional, no obedece a rendimientos de desarrollo interno, sino que la economía nacional se ubica en el sector. La naturaleza de esta concentración, anuncia que a mayor expansión del sub sector tradicional, la productividad de los sectores tenderá a disminuir, acarrean- do estancamiento.

Sobre el comportamiento del sector terciario en el Estado de México, se ha encontrado para la década de los ochenta: Primero, que la terciarización de la economía mexiquense presenta un dinamismo cuya estructura interna 
es predominantemente tradicional. Segundo, los municipios metropolitanos, donde la estructura es diversificada ésta se encuentra fuertemente condicionada a la actividad industrial y determinado por el nivel de ingreso de la población. Tercero, en términos espaciales se dice que al mismo tiempo que sucede la concentración del empleo en actividades terciarias tradicionales se provoca concentración espacial en los municipios metropolitanos (Hoyos, 1995).

La concentración sectorial y espacial es una situación compleja de orden estructural. Generalmente la expansión de las actividades económicas están asociadas, entre otras razones, a las aglomeraciones urbano industriales. En este contexto, en los países como México, predomina la tradicionalización del empleo urbano pues los sectores llamados dinámicos afrontan contracción y crisis.

Sobre los municipios metropolitanos que comandan la dinámica económica, social y política de la entidad, es importante saber si en la nueva modernización, podrán jugar un papel integrador y lograr organizar espacialmente la actividad interna. Desde la lente del análisis regional, ${ }^{1}$ aquí nos centraremos en la distribución y evolución del sector en los considerados municipios metropolitanos. Se presenta primero, en forma breve, algunas explicaciones dadas al crecimiento del sector. Segundo, se reflexiona en la naturaleza económica de la concentración metropolitana. Luego, se presenta un programa descriptivo, tanto de la fuerza de trabajo como de la demanda del sector. Para cerrar el artículo se hacen algunos comentarios.

\section{Sector Terciario. Expansión y reestructuración}

Respecto a los cambios en la estructura del empleo en los servicios, todavía no existen explicaciones consolidadas. Si embargo es frecuente encontrar en la literatura algunos conceptos que brindan posibles caminos de su entendimiento; algunos autores han denominado la era de la "desindustrialización" (Cairncross, 1982), sugieren que se trata del cambio de una sociedad industrial por otra "postindustrial"( Bell, 1989), que la influencia de la investigación, tecnología e información han propiciado la "revolución de los servicios" (De Mateo y Carner, 1988), y se distingue la formación de un sector económico superior llamado "cuaternario"(Gentilicci, 1989).

1 La ciencia regional, como todas las ciencias sociales, se ocupa del estudio del hombre y su interacción y ajustes continuos con su entorno físico. No obstante, la ciencia regional se auto limita al estudio de problemas para los cuales es fundamental el enfoque de un espacio o región. Concierne a investigación y estudio de la estructura, función y operación de regiones desde un punto de vista económico, social y político” (Cfr. Isard. W. y M. Chatterji, 1993) 
La rápida expansión, diversificación y especialización de los servicios ha tomado lugar desde 1945 en todo el mundo. Hay notables diferencias en la propagación de actividades en los diferentes países y entre regiones desarrolladas y menos desarrolladas. Estas diferencias son extensivas a los contrastes estructurales. Los servicios nuevos desarrollados y especializados, tales como finanzas y bancos, comercialización y difusión son importantes en las regiones desarrolladas, mientras que en las menos desarrolladas los servicios "viejos" y tradicionales, tales como el transporte, el comercio al detalle y distribución comprenden la mayor parte del sector servicios (Daniels, 1985).

La modernización tecnológica predominante, adquiere formas particulares entre las estructuras de las economías nacionales, grado y tipo de desarrollo histórico marcan las características distintivas de la terciarización. En este fenómeno ocurren dos procesos simultáneos: reestructuración de los procesos generadores de servicios modernos productivos que se componen de organización y conocimiento, bajo empleo, ingreso alto, calificación educativa especializada y; conformación de dos subtipos de actividad económica; uno de ellos con crecimiento de empleo con baja incidencia del producto nacional, salarios bajos y otro, denominado informal. ${ }^{2}$

El subsector moderno produce y difunde información, nuevas formas de organización, administración, profesionalización y planifica los requerimientos de los nuevos procesos productivos en el ámbito de las empresas. En el subsector nomoderno, se ubican las actividades destinadas al consumo final, se asocian generalmente con baja productividad, alta ocupación, bajos salarios, por tanto, baja incidencia relativa en el producto interno. Éstas responden a la dinámica socioeconómica en general.

Los servicios al productor, en .los países desarrollados, han crecido y rápidamente se han diversificado En contraste con los servicios al consumidor, la localización de los servicios al productor son dominados por la centralización en un pequeño número de centros con mayor empleo, los que ofrecen un rango de aglomeración y economías de urbanización que son prerrequisitos para la provisión efectiva y especialización de los productos de tales servicios.

Las explicaciones que se han dado al incremento de la demanda de los servicios al productor, incluyen a las oportunidades que ofrecen las nuevas

2 La OIT en 1972 acuñó el concepto. A la fecha existen diversos enfoques y conceptualizaciones. Aquí se presenta la definición del PREALC: el sector informal "concentra a los ocupados que normalmente no ejercen su actividad en las empresas organizadas y constituye el resultado visible del excedente relativo de la fuerza de trabajo. En este sector se incluye a la mayor parte de los trabajadores por cuenta propia; el servicio doméstico y los ocupados (patrones o empleados) en empresas pequeñas no organizadas formalmente" (Cfr. Tokman, 1995). 
tecnologías en los cambios de la estructura Y sobre el tamaño de la demanda que ellas mismas proporcionan desde fuera más que interno a las empresas. Las grandes pueden plantear sus requerimientos a través de una transferencia de bienes y servicios, más que concentrar la mano de obra con alta destreza pero en situaciones ocasionales. Así las empresas que brindan servicios al productor, en cambio, pueden proporcionar un mismo producto a un amplio rango de clientes a un costo unitario más bajo. Con ello una organización grande puede reducir el número de jefes administrativos y simplificar equipo de directores (Daniels, 1985). .

La globalización de las finanzas y la tecnología, define nuevas formas de organización del territorio donde surge el concepto de reestructuración del territorio. Aquí el papel de las regiones dinámicas es el de la especialización funcional; activar las funciones con mayores posibilidades de competencia nacional e internacional.

La reestructuración del territorio, en países desarrollados, es radial con un centro como potencia económica dominante, mano de obra bien formada, organización del trabajo, tecnología avanzada y acuerdos sociales reglamentados. A medida que se deja el centro existen condiciones con mayor flexibilidad y mano de obra de menor calificación y salarios más bajos. Sin que ello provoque una deslocalización de las empresas del centro a la periferia. Se forma así, una especie de división interregional del trabajo, en la cual las industrias más complejas, mismas que demandan mayor implicación y calificación de los asalariados, están en el centro y las industrias más flexibles, más banales que requieren mano de obra poco calificada y que por tanto pueden ser flexibles, se encuentran en la periferia (Pradilla y Lipietz, 1993).

Lo anterior permite decir que la organización regional a luz de la globalización y reestructuración productiva, ${ }^{3}$ opera en la heterogeneidad de las estructuras internas. Para el caso de la Comunidad Económica Europea, refleja una distribución en círculos concéntricos: la mayor rigidez en salarios/ horas/hombre, salarios/mano de obra calificada, procesos técnicos más modernos, ramas y subramas especializadas, política social menos laxa, política eco lógica más rigurosa, varían del centro a la periferia, lo cual indica que los modelos de organización y producción "tayloristas", "fordístas" y de "flexibi-

3 "De la actual reestructuración productiva capitalista se puede decir esquemáticamente lo siguiente: la caída de la tasa de ganancia y los costos crecientes del dinero explican en gran medida la necesidad de reducir el tiempo de rotación del capital, lo cual se logra con la innovación tecnológica, dentro de la cual debemos incluir también las innovaciones en materia de administración y organización de la producción y circulación capitalista”

(Zaragoza, 1993). 
lidad" no se excluyen ni técnica ni espacialmente, éstos se aplican en función de las posibilidades de integración y reestructuración, surgen así políticas y reglamentaciones diferenciadas.

Dicha organización económica con integración especializada diferenciada reporta ya consecuencias no muy halagüeñas. "La experiencia española en los años posteriores a su ingreso a la CE es muy ilustrativa. Si bien la demanda externa explica parcialmente el aumento del empleo en las manufacturas, los sectores 'intensivos en fuerza de trabajo' registran los mayores retrocesos en cuanto a empleo derivado de la demanda externa” (Rendón y Salas, 1993). Por otra parte, la variación espacial del nivel industrial ha propiciado que las telecomunicaciones, distribución de las casas matrices de dirección nacional e internacional de bancos, hospitales y servicios médicos se reorganicen espacialmente (Daniels, 1985).

La terciarización de las economías en general, podría entenderse como aquellos cambios por los que actualmente transitan los procesos productivos consecuencia del desarrollo técnico científico, que favorecen el surgimiento de servicios modernos con importante productividad. Cambios en la calidad del empleo. Cambios que provocan encadenamientos de alta tecnología entre sub sectores modernos de la economía. Cambios que modifican la composición de los factores de producción de bienes y de servicios. Cambios que modifican los estándares del consumo en la población.

Los procesos de modernización que surgen en los países industrializados, se han difundido rápidamente en las economías nacionales. A este respecto R. Dambois y L. Pries (1993), hacen una distinción importante: “..., no se difunden de la misma manera y al mismo ritmo entre y dentro países diferentes, sino en procesos complejos de búsqueda, selección, aplicación y adaptación según los contextos económico - sociales particulares". En el caso particular de los cambios tecnológicos en México, para 1987 se encontró que $67 \%$ de las empresas industriales tenían tecnología artesanal; que 25\% estaba mecanizada, aunque obsoleta y que sólo $8 \%$ poseía tecnologías modernas (Flores, 1990, citado por Bizberg, et.al., 1993). Ello acota el ámbito de los cambios y las características concentradas de las innovaciones.

En México, el crecimiento del sector puede estar asociado al proceso de modernización de la economía, puede ser en parte consecuencia del modelo industrial. Este modelo impulsa subsectores y ramas susceptibles de competencia exterior, y del otro lado, la fuerza de trabajo se ubica en proporciones importantes en sectores considerados "no Competitivos". Esto es, el sector se expande como consecuencia de la caída de la demanda de productos manufacturados, por la contracción de la demanda de empleo global y en menor medida por los cambios tecnológicos dentro del mismo. 
Ello nos sitúa en un fenómeno de concentración estructural compleja, que plantea retos de ordenamiento territorial, de política económica, social, de regulación administrativa hacendaria y desafíos analíticos. La terciarización es un fenómeno concentrador excluyente. Los procesos económicos dinámicos son excluyentes porque conforman un sub- sector terciario moderno en relación a la eficiencia y productividad. Los procesos son concluyentes porque las actividades y la población ubicada en el sector se concentran geográficamente.

Por su parte, las interrelaciones económicas entre los subsectores dinámicos de la industria manufacturera y servicios aumentan la complejidad del manejo económico y espacial de los recursos en general. Las actividades se estimulan económicamente unas a otras, surgen y se desarrollan donde son posibles las economías de aglomeración. La aglomeración urbana se refiere a la reunión de actividades económicas y población en las ciudades. Esto es así, de acuerdo con el análisis espacial de la economía, porque las empresas, la población y el gobierno obtienen beneficios de la proximidad física, pero son diferenciados.

Por múltiples procesos, las actividades terciarias se relacionan con la industrialización, urbanización, centralización administrativa, entre otros fenómenos. Cada uno de éstos ejerce influencia entre ellos determinando el comportamiento y la estructura del sector. Ésta reacciona en relación con los cambios que ocurren en cada uno de los procesos, causando lo que denominamos círculos de influencia. Las actividades crecen conforme al nivel del ingreso y de acuerdo con el avance de la urbanización, donde los cambios según sean integradores o excluyentes impactarán un círculo hacia el desarrollo o un círculo hacia el declive. En condiciones de crecimiento y reestructuración productiva extensiva, se dinamizan principalmente las actividades modernas. En un proceso recesivo incrementan las actividades tradicionales y el sector informal. Esto es, las características del tipo de terciarización cambian con las innovaciones tecnológicas, procesos técnicos, inversión, nivel de ingreso, profesionalización del trabajo y crecimiento poblacional.

En el actual debate regional se dice que, "las regiones que ganan" son las urbanas, las fábricas y las oficinas vuelve las grandes ciudades es decir a las megalópolis, donde la especificidad de la ciudad es la especialidad terciaria. "La distinción 'industrial/terciaria' no es tan evidente (en el contexto del progreso de la telemática). Se trata de actividades complementarias; y las 'regiones que ganan' son ante todo regiones productoras de bienes de exportación, es decir, de 'bienes manufacturados' o de servicios facturables" (Benko y Lipietz, 1991). 
Una reflexión optimista para América Latina, en el marco de los procesos de regionalización global, es la de S. Boisier (1994): “Tal vez ha llegado el momento de reconocer que los intentos dirigistas y del centro hacia abajo para configurar regiones mediante el artificio de sumar y agregar unidades de la división política-administrativa, han llegado a un callejón sin salida. Las resistencias son grandes y los entrabamientos pueden hacer perder oportunidades - de inversión, de mercados, de asociación-: aprovechar oportunidades es la única fórmula de triunfo en un mundo competitivo. La competencia no es el sistema que permite triunfar siempre a los más fuertes, sino el sistema que permite el triunfo sistemático de quienes saben aprovechar las oportunidades, es decir, de los más hábiles".

La aproximación analítica que subyace en este trabajo, es aquella de dimensionar las "bondades y alcances del mercado", pues se trata de crecimiento económico con profundización de la concentración de mercados e ingresos. En la globalización, los flujos de innovación, las reestructuras tecnológicas e inversiones se establecen entre ramas modernas de las aglomeraciones urbanas dinámicas, definiendo dos procesos económico-espaciales: el primero se desarrolla dentro de un movimiento más general, importante y desarrollado, que excluye a los países subdesarrollados (cuando hablamos a nivel internacional) y ciertas regiones (a nivel subnacional) y; el segundo se lleva a cabo dentro de un movimiento particular, en relación a las condiciones internas. Es decir, que la reestructuración económica implica integración subnacional diferencial y segmentada. Para el caso mexicano, los "polos de desarrollo" de los sesenta, que han propiciado regiones urbano-industriales, son los que reportan diversidad y relativo dinamismo. Se convierten en los principales o únicas regiones que brindan cierta especialización con posibilidad de integración a los procesos modernos de reestructuración productiva.

\section{Terciarización metropolitana}

La concentración de actividad económica y del proceso de urbanización de la población, son fenómenos que se presentan simultáneamente, ello ha derivado en la constitución de un sistema nacional de aglomeraciones metropolitanas. La expansión del sector terciario se asocia a estos procesos, a los impactos de los adelantos tecnológicos, la concentración demográfica y condiciones de la planta industrial.

Las transformaciones sectoriales del empleo nacional y regional modifican las actividades del sector terciario e inciden en las características económicas del proceso de metropolización. La "sobreterciarización" de las zonas 
urbanas puede ser un hecho eventual y eminente. Ello implica la caída de la productividad del sector, pues se trata de una misma proporción o caída del PIB terciario (en forma prolongada) para un mayor número de población ocupada en el sector.

Los modelos de desarrollo económicos seguidos en México, han venido consolidando los elementos componentes de la concentración que actualmente se observa en todos los planos de la vida nacional. La centralización política y la concentración de las actividades económicas; el crecimiento industrial, la comercialización, la administración, la actividad financiera y la oferta de mejores condiciones del nivel de vida, son factores que han formado regiones geográficas. El “centro" de naturaleza político-económica y de alcance nacional es la región central, más específicamente la ciudad de México y su emplazamiento territorial metropolitano. Es casi generalizado que en los países de Latino América alta concentración coincida con la capital política administrativa.

A lo largo del proceso de desarrollo económico mexicano, los cambios sucesivos de modernidad del presente siglo, los mejores rendimientos crecientes de la economía y de la acción política social se han dado en los centros urbanos mayores. Donde las políticas de inducción-concentración combinadas con las de difusión del crecimiento económico revelan que históricamente han dominado los efectos de atracción, polarizando el desarrollo. Actualmente el dilema para todos los sectores de la administración, es la concentración compleja y las disparidades regionales.

Las políticas que hablan de la distribución territorial de la actividad económica y la población, se enfrentan a la lógica de la economía y la imposibilidad de revertir las inercias. A este respecto señala J. Delgado (1994), que conceptos "expansión descentralizada", "suburbanización deslocalizada" y el de "concentración ampliada" designan el mismo proceso: crecientes oleadas de población y actividades desde el centro hacia la periferia; el primero pone el acento en la descentralización, el segundo, en lo que tiene de azar la elección periférica, mientras que el tercero busca subrayar que es el centro quien expande su influencia.

La región central de México, es un claro ejemplo de los procesos nacionales. En ella se asienta una proporción importante de la redistribución de población, genera la mayor parte del producto nacional. Asimismo, se convierte en el ámbito del fenómeno de terciarización. Se observan los cambios de la estructura del sector terciario: consecuencias de política económicas, apertura de mercado, reorganización de procesos productivos y; reacomodos geográficos del ordenamiento territorial nacional. 
La región central para 1990, concentra arriba de 27 millones de habitantes y algo más de 4 millones de población económicamente activa en el sector terciario, es decir, con- centra una tercera parte de la población nacional y por arriba de la tercera parte de PEA terciaria (33 y 40 por ciento respectivamente). Específicamente en el Distrito Federal y Estado de México viven 66 por ciento de la población regional y 22 por ciento del país. El sector terciario participa aproximadamente con 79 por ciento en la región y 31 por ciento en el país (Hoyos, 1995).

El constante crecimiento demográfico y expansión física del Distrito Federal rebasó los límites jurídicos con el Estado de México allá por las años cincuenta y la ciudad de México empezó a incorporar municipios mexiquenses . De esta forma la entidad comparte atributos con la ciudad capital: participa de la concentración de la actividad industrial y de población en los municipios denominados metropolitanos. Posteriormente, en la Capital del Estado, la ciudad de Toluca, se conforma una zona metropolitana propia, ligada funcionalmente a la primera. Este proceso dio por resultado alta concentración a su vez alta dispersión. Situación que se observa en la configuración territorial de la dinámica económica y demográfica estatal. Se constituyen así dos zonas de mayor dinamismo.

La primera, Zona Metropolitana de la Ciudad de México (ZMCM), refleja cambios de alcance nacional, tanto porque alberga la capital política del país, el Distrito Federal, como por los modelos políticos y económicos que en ella han centralizado Y concentrado los procesos. Los municipios mexiquenses de la Zona, ${ }^{4}$ están asociados a la sinergia económica y ejercen atracción comandando efectos de orden primario en la redistribución territorial nacional.

La segunda, Zona Metropolitana de la Ciudad de Toluca (ZMCT), ${ }^{5}$ presenta el componente de capital política de la entidad y el dinamismo económico alcanza aproximadamente al Valle de Toluca. La cercanía geográfica con la primera, ha permitido que los vínculos económicos sean intensos y que ésta presente una forma física en correspondencia a los ejes carreteros que las comunican, al mismo tiempo que define las interrelaciones de orden

$4 \mathrm{La}$ ZMCM consta de 16 delegaciones y existen discrepancias en el número de municipios mexiquenses integrados para 1990: según Garza (1993) son 27; de acuerdo con Negrete (1995) son 29 y para Sobrino (1992) son 26 los municipios.

5 La ZMCT, de acuerdo a los criterios de estudio, varía el número de municipios que la integran y no necesariamente se trata de los mismos: para el "Plan Regional Metropolitano de Toluca” (1993) consta de siete, según Garza (1993) son seis, para Negrete (1995) e INEGI (1990) son cinco y Sobrino (1995) sostiene que son siete 
menor. Las dos zonas presentan dinámica y estructura propia de orden diferenciado pero articulados.

El tipo de crecimiento económico experimentado por la entidad durante los últimos cincuenta años, ha consolidado los desequilibrios regionales; los recursos humanos y la estructura productiva siguen la inercia concentradora. Los municipios más atractivos para la inversión en servicios comerciales, servicios de mejor calidad y relativamente modernos son aquellos que reportan mayor crecimiento poblacional con importante proceso de metropolización, en algunos casos transitando hacia procesos megalopolitanos y además con importante desarrollo industrial. Es decir allá donde existe concentración y diversidad de mercados para obtener ventajas económicas relativas.

La diversificación de los centros metropolitanos puede permitir la especialización, lo mismo que las comunicaciones, los flujos de información y nuevas formas de organización de las empresas, se sobreponen a los beneficios de la proximidad física de la economía (etapa de la localización clásica). La existencia de una estructura interna especializada y flexible -tecnológica y organizacional- garantiza la exportación regional y el comercio internacional; exigencia de la apertura de los mercados.

Tal como se señala: "Además del estímulo que la concentración de las condiciones generales de la producción representa para las manufacturas, la aglomeración espacial de las actividades industriales obedece también a la necesidad de utilizar una gran diversidad de servicios especializados y más tecnificados, que van cobrando autonomía y separándose paulatinamente del ámbito propiamente fabril. Resulta, pues que los servicios destinados al productor se desarrollan concentradamente en torno a los núcleos de mayor dinamismo industrial. Para 1980, la ciudad de México aglutinaba el 48\% del valor generado por los servicios al productor, mientras que su participación en el empleo y el número de establecimientos correspondía al $42.4 \%$ y 35.3\%, respectivamente" (Garza y Rivera, 1993).

La relación entre aglomeración y dinamismo del subsector terciario moderno, exhibe al menos dos situaciones: fuerte dependencia de la diversidad y cambios técnicos organizativos de las empresas manufactureras y; alta concentración demográfica, de mercados industriales, de consumidores que en conjunto permiten la especialización. Para el caso de México esta situación agrava la perpetuidad de la primacía de las regiones dinámicas.

En un estudio de las zonas metropolitanas mexicanas de los ochenta, se encontró que los fenómenos de terciarización y relocalización del empleo industrial hacia las periferias metropolitanas son los cambios sobresalientes 
que experimentó la estructura sectorial y territorial del empleo metropolitano. "La estructura del empleo y las funciones económicas de la ciudad son elementos clave en la explicación de la evolución metropolitana. Los mercados de trabajo urbanos y metropolitanos ocupan proporciones cada vez más importantes de la población económicamente activa del país y en ellos se refleja especialmente el impacto de los avances tecnológicos en la producción" (Negrete y Salazar, 1986)

Sobre las actividades económicas y el empleo, específicamente en el Área Metropolitana de la ciudad de México se ha constatado que “...en los años ochenta se acentuaron tres fenómenos importantes que habían aparecido en la década previa: 1. La pérdida de la capacidad relativa del sector manufacturero de generar nuevas ocupaciones. 2. El freno a la proletarización de fuerza de trabajo. 3. La terciarización del mercado de trabajo se profundiza aún más. Estos procesos están estrechamente vinculados entre sí, porque el ritmo al que crece el empleo en las manufacturas tiene fuerte impacto sobre evolución del empleo asalariado global. Esto se debe al hecho de que en este sector la inmensa mayoría de los trabajadores (92\%) realiza su actividad a cambio de un salario. Mientras que en los dos restantes el peso relativo del trabajo no asalariado es todavía muy importante, particularmente en el comercio (46\%)" (Salas, 1992).

En el actual contexto de la urbanización de la población, etapa de aglomeraciones metropolitanas, las empresas consideran conveniente localizarse principalmente en las ciudades más grandes del sistema nacional, donde encuentra posibilidades de realizar interrelaciones de compraventa ente empresas. El desarrollo económico y social de las regiones debe ahora ser analíticamente situado en la reestructuración productiva, para en ellas dimensionar la dinámica de la terciarización.

Las zonas metropolitanas, en la actual coyuntura económica nacional, concentran fuerza de trabajo y padecen contracción del empleo en general; revelan transferencia de fuerza de trabajo del sector manufacturero hacia otros sectores de la economía, al mismo tiempo en éstas residen todo tipo de trabajadores y ocupaciones, se localiza el capital, la inversión, surgen innovaciones y la especialización de las funciones. Lo que en ellas ocurre es un fenómeno interrelacionado entre la dinámica local y la nacional. En este proceso es clara la relación entre dinámica territorial y economía nacional. Es decir, son el lugar geográfico donde evolucionan la actividad y la población. 


\section{Municipios Metropolitanos. Oferta y demanda de empleo del sector terciario}

Los 37 municipios considerados metropolitanos para 1990 concentran 79 por ciento de la población mexiquense, poco más de 7 millones y medio de habitantes. De este total, 89 por ciento corresponde a la ZMCM y 11 por ciento a la ZMCT. Es decir, que en el 30 por ciento de los municipios vive la mayor parte de la población de la entidad. Contexto de alta concentración donde se analizará la estructura eco- nómica municipal, lo relativo a la oferta de fuerza de trabajo y la demanda del sector terciario.

\section{a) Oferta de fuerza de trabajo}

El proceso de transferencia de la fuerza de trabajo, de los sectores agropecuario y manufacturero al terciario en los municipios, se observa a través de la participación relativa de la PEA terciaria en el total del empleo. Se considera oferta, porque la población manifiesta haber trabajado o realiza algún trabajo en forma ocasional, periódica o permanentemente en: a) comercio, restaurantes y hoteles; b) transporte, almacenamiento y comunicaciones; c) establecimientos financieros, seguros, etc.; o en los d) servicios comunales, sociales y personales.

De acuerdo con las tasas de crecimiento porcentual promedio de la PEA, la proporción del sector presenta variaciones en los últimos 30 años. El conjunto de los municipios reportan incrementos, lo que denominamos terciarización. La dinámica del sector es heterogénea y fluctúa en el periodo: Los primeros 20 años, se puede considerar etapa de alta terciarización, en general los municipios en estudio exhiben incrementos (excepto Nextlalpan). En cambio en los años ochenta, es significativa la disminución e incluso el sector pierde empleo sectorial municipal. No obstante, podemos decir que el sector ha crecido de manera constante en las décadas analizadas, (por ejemplo, la tasa promedio de 1960 - 1990, va de 5.58 por ciento para San Mateo Atenco a 0.15 por ciento para Cuautitlán Izcalli), pero al interior y entre los municipios operan situaciones económicas y demográficas que propician tal situación (Cuadro 1).

La redistribución intersectorial de la fuerza de trabajo que favorece el incremento del sector, puede estar asociado al propio crecimiento natural de la población en edad de trabajar, a las exigencias de cualificación de los trabajadores que debido a la no correspondencia se ubican en ocupaciones con baja calificación y puede explicarse por la contracción de la demanda de empleo global. 


\section{b) Demanda ocupacional del sector terciario, 1993}

La actividad económica nacional para 1993 se realiza en poco más de 2 millones de establecimientos económicos, mismos que captan arriba de 12 millones de trabajadores. La mayor aportación de empleo lo realizan los servicios (44.41\%), la Industria manufacturera (27\%) y el comercio $(25.05 \%)$. En este año, los establecimientos comerciales son dominantes $(52.65 \%)$, le siguen los de servicios (34.01\%) y luego la manufactura (11.72\%).

Es decir, según el último censo económico, los sectores comercio y servicios demandan 69.46 por ciento de la ocupación total (Cuadro 2).

El Estado de México concentra aproximadamente 10 por ciento de la demanda de empleo global nacional. En cuanto a la demanda por sectores, la entidad capta 13.03 por ciento en el industrial 9.88 por ciento en el comercio y 8.35 por ciento en los servicios a nivel nacional. Al interior del estado, la actividad económica se comporta semejante al agregado nacional: los servicios, la manufactura y el comercio son los sectores con mayor demanda. Pero de acuerdo con la estructura ocupacional, es importante la captación que realizan en conjunto el comercio y los servicios, ello significa 62.46 por ciento de la entidad (Cuadro 2).

Los municipios metropolitanos mexiquenses, concentran 90.22 por ciento de la demanda de empleo y 87.37 por ciento de las unidades económicas censadas del total del sector terciario de la entidad. Los municipios de la ZMCT, demandan 13.07 pro ciento del empleo en 11.12 por ciento de los establecimientos. La ZMCM, por su parte, capta 77.16 por ciento del empleo en 76.25 por ciento de los establecimientos. Sin embargo, del conjunto de los 37 municipios, destacan en forma importante en actividad terciaria, Naucalpan, Tlalnepantla, Ecatepec, Nezahualcóyotl y Toluca (Cuadro 3).

La estructura interna del sector terciario de la entidad revela dominancia del comercio, éste capta 59.14 por ciento, en cambio los servicios significan 40.86 por ciento del empleo generado en el sector. Entre los municipios metropolitanos, también dominan las actividades comerciales, destacan San Mateo Atenco y Zinacantepec en la ZMCT, Chiconcuac y Teoloyucan en la ZMCM. La proporción de empleo en los servicios es mayor sólo en el Municipio de Ocoyoacac para la ZMCT y Tepotzotlán y Huixquilucan para la ZMCM. Por otro lado, existen casos en los cuales el empleo terciario es relativamente balanceado para los subsectores, tales como Metepec y Lerma en la ZMCT, Naucalpan, Tlalnepantla y Atizapan de Zaragoza en la ZMCM (Cuadro 3).

De lo arriba descrito se resume que la actividad económica de la entidad para 1993, se basa en el sector terciario, más específicamente en el comercio. Además que ésta se asienta en contados municipios metropolitanos. 
Por las limitaciones de la información disponible (Resultados Oportunos de los Censos Económicos 1994), no se realiza un estudio comparativo con los años anteriores, ni se aborda por ramas o clases de actividad. No obstante, aunque en forma aproximada, es significativa la concentración económica y espacial.

Por la naturaleza de los cambios técnicos productivos y los diferenciales de desarrollo, los procesos territoriales derivados de la globalización no serán generalizados para sectores y ramas de las regiones dinámicas, ni del conjunto de las economías urbanas, se presenta en segmentos de la economía y en porciones del emplazamiento de las ciudades. Se trata de un proceso de formación de estructuras económica-espacial diferenciadas pero articuladas entre si. Sólo ciertas actividades económicas modernas de algunas zonas urbanas podrán alcanzar las exigencias de la economía internacional; especializar para exportar. Todo ello, en un marco donde las economías de las zonas urbanas padecen rezago económico y de atención social; crecimiento de la fuerza de trabajo sin posibilidad de inserción ocupacional con ello decrece la población asalariada e incrementan las actividades de subsistencia, fenómeno que se agudiza en las regiones urbanas de hoy. Estas presentarán crecimiento social de población, se incorporará más suelo para uso urbano, se observarán cambios en la estructura física y transformaciones diferenciadas en la imagen urbana.

En el marco de la urbanización terciaria mexicana y específicamente para el Estado de México, parece ser que el sector no sólo se concentra por la relativa dinámica de la industria manufacturera, en cambio la diversificación de actividades económicas en general hacen suponer la relación entre subsector especializado moderno, procesos técnicos, con la concentración de mercados de consumidores, ello debido a las ventajas que se obtiene de la variedad de mercados locales. Los servicios se concentran porque están especializados y forman parte de eslabonamientos internos y externos de las empresas, pero sobre todo porque atienden a un sin número de mercados de consumo y a una gama de empresas.

Economía abierta y crisis económica nacional, ${ }^{6}$ marco en el cual las estructuras de empleo y las condiciones productivas de los municipios metro-

6 De la coyuntura nacional, se puede mencionar tres aspectos, entre otros: primero, imposibilidad inmediata de competencia externa debido a i) readecuaciones técnicas y organizacionales de las empresas; ii) altos costos totales de producción para la exportación y iii) baja flexibilidad del producto. Segundo, crisis económica interna; por un lado se encuentran severamente afectados la planta productiva, el empleo y los ingresos, y del otro, fragilidad del sistema financiero. Tercero, en lo social, atmósfera política poco estable, aumento de riesgos políticos y de inseguridad pública. 
politanos mexiquenses, se enfrentan a una dinámica contradictoria: diversificación-especialización y diversificación -tradicionalización de la economía. De ellas, la segunda relación puede ser predominante por su alta dependencia a los mercados locales, concentración de población e ingresos. La economía se desplaza hacia segmentos de actividades económicas flexibles y competitivas, capta segmentos calificados y polivalentes de fuerza de trabajo, cambia de espacios de comercialización, todo ello en las aglomeraciones mayores, como mecanismo de reproducción. Así la terciarización continuará complejizando la situación económica y social de las zonas metropolitanas.

\section{Comentarios finales}

Hasta el momento, ni los tomadores de decisiones ni los analistas le han prestado atención a la terciarización de la economía y sus implicaciones inmediatas sobre la población y el territorio. Actualmente se menciona mucho, se indaga poco.

Es un sector con importante participación en la economía mexicana, como tal adolece de estudio, específicamente por parte de los economistas y estudiosos de la economía urbana. Los economistas argumentan que es un sector muy heterogéneo, que debido a la información con que se cuenta, su estudio no puede ser más que agregado y general. Preferentemente se estudia la comercialización internacional de los servicios; los financieros, el transporte y las comunicaciones. Por otro lado, hay un grupo de investigaciones sobre empleo sectorial y éste no es el centro de discusión, aparece cuando hablan de la multiplicación de los micro establecimientos, marginalidad y economía informal.

De igual manera el sector es escasamente analizado en la relación que guarda con la configuración territorial: desde el ámbito de los estudios regional urbanos, en el contexto de la economía urbana y más escaso aún en el sistema de ciudades metropolitanas. Las investigaciones con las que contamos estudian la "centralidad" de las actividades urbanas, la distribución de las funciones económicas en los centros urbanos, sobre los servicios públicos y es mínima la que corresponde al sector servicios. Los analistas de esta disciplina argumentan que una explicación de la economía terciaria urbana, vendría del estudio desagregado de las funciones económicas, porque el contexto en que se desempeñan es complejo y de múltiples aristas.

Los argumentos anteriores vienen postergando el conocimiento de la naturaleza estructural de la economía nacional-urbana, en el largo camino de la concentración económica-espacial, fenómeno que ha derivado en altos 
costos sociales. Es un espacio abierto al análisis en el contexto de la investigación en México: en el marco de la concentración estructural compleja, en el rezago conceptual y condiciones de la información, el fenómeno de la terciarización aguarda atención.

\section{Bibliografía}

Bell, Daniel, 1989: El Advenimiento de la Sociedad Post industrial, Madrid: Alianza Universidad.

Benko, G. y A Lipietz, 1991: 'Posiciones en el nuevo debate regional' Traducción realizada por Ramírez B, del libro Les Regiones Qui Gagnent-Districts Et Réseaux: Les Neuveaux Paradigmes de la Geographie Industrielle. Paris: Presses Universitaires de France.

Boisier, S., 1994: “Crisis y alterativas en los procesos de regionalización” en ONU Revista de la CEPAL 52, abril, Santiago de Chile: CEPAL.

Bizberg, I., Enrique de la Garza y Yolanda Montiel, 1993: "Modernización empresarial y relaciones industriales en México" en Dombois, R. y Pries, L, Modernización Empresarial: Tendencias en América Latina y Europa. Venezuela: Fundación Friedrich Ebert de Colombia, FESCOL.

Daniels, P.W., 1985: Service Industries. a Geographical Appraisal. Mathuen.

De Mateo F. y Carner, F., 1988: "El sector servicios en México: un diagnóstico preliminar" en COMERCIO EXTERIOR Vol. 38 N.1

Delgado, J., 1994 “La concentración ampliada de la dudad de México", mimeo. México: Instituto Mora.

Dombois, R. y L. Pries, 1993: Modernización Empresarial: Tendencias en América Latina y Europa. Venezuela: Fundación Friedrich Ebert de Colombia, FESCOL.

Garza, G. y S. Rivera, 1993: “Desarrollo económico y distribución de la población urbana en México, 1960 - 1990” en Revista Mexicana de Sociología, IIS /1/93 Año LV, Núm. 1, enero-marzo 1993, México: UNAM.

Gentilicci, E. Grasso y A. Samperi, G., 1989: "Commercio e Structura della Rete Terziaria in Scilia una Esperienza di Analisl.' Giornate de Studio Analisi Satistica de Dati Territoriali., mimeo Bari, Italia.

Gobierno del Estado de México, "Plan Regional metropolitano de Toluca" en Gaceta de Gobierno, Núm. 52, Tomo CLV, 18 de marzo de 1993. Toluca: GEM

Hoyos C., G., 1995: Estructura y Distribución Territorial de la Terciarización del Estado de México, Tesis de Maestría: Colegio Mexiquense, A. C. 
Isard, W. y M. Chatteriji, 1993: "Ciencia regional: nuevo orden mundial y el desarrollo de México en la era del TLC” en Problemas del Desarrollo, Vol. XXIV, núm. 93, abril-junio. México: IIEc-UNAM.

Lever, W.F., 1991: "Deindustrialization and the Reality of the Post-industrial City", in Urban Studies, Vol. 28, núm. 6, pp.983-999

Negrete S, M.E. y S. H. Salazar, 1986: “Zonas Metropolitanas, 1980” en Estudios Demográficos y Urbanos, Vol. 1, núm. 1. México: COLMEX / CEDDU.

Negrete S, M.E., 1995: "Evolución de las zonas metropolitanas en México" en Garrocho, C. y L. Jaime Sobrino, coords. Sistemas Metropolitanos. Nuevos Enfoques y Prospectiva, Zinacantepec: El Colegio Mexiquense A.C. y SEDESOL.

Pradilla C. yA. Lipietz, 1993: "Globalización, posfordismo y territorios, entrevista con Alain Lipietz”, en CIUDADES núm. 20, octubre-diciembre 1993. México: RNIU.

Rendó, T. y C. Salas, 1993: "EI empleo en México en los ochenta: tendencias y cambios” en Comercio Exterior, Vol.43, núm. 8, agosto 1993, México.

Salas P., C., 1992: "Actividad económica y empleo en el Área Metropolitana de la Ciudad de México: 1979-1990”, en La Zona Metropolitana de la Ciudad De México. Problemática Actual y Perspectivas Demográficas y Urbanas. México: CONAPO.

Sobrino, Luis Jaime, 1992: "Estructura ocupacional del sector servicios en la Ciudad de México, 1960-1988”. En La Zona Metropolitana de la Ciudad de México. Problemática Actual y Perspectivas Demográficas y Urbanas. México: CONAPO.

Tokman, V., 1995: Comp. El Sector Informal en América Latina. Dos Décadas de Análisis. México: Consejo Nacional para la Cultura y las Artes.

Zaragoza, J., 1993: "EI papel de los servicios en la reestructuración industria"” en Problemas del Desarrollo, Vol. XXIV núm. 95, octubre-diciembre. México: IIEc-UNAM. 
Convergencia Revista de Ciencias Sociales, núm. 12/13, 1996, Universidad Autónoma del Estado de México

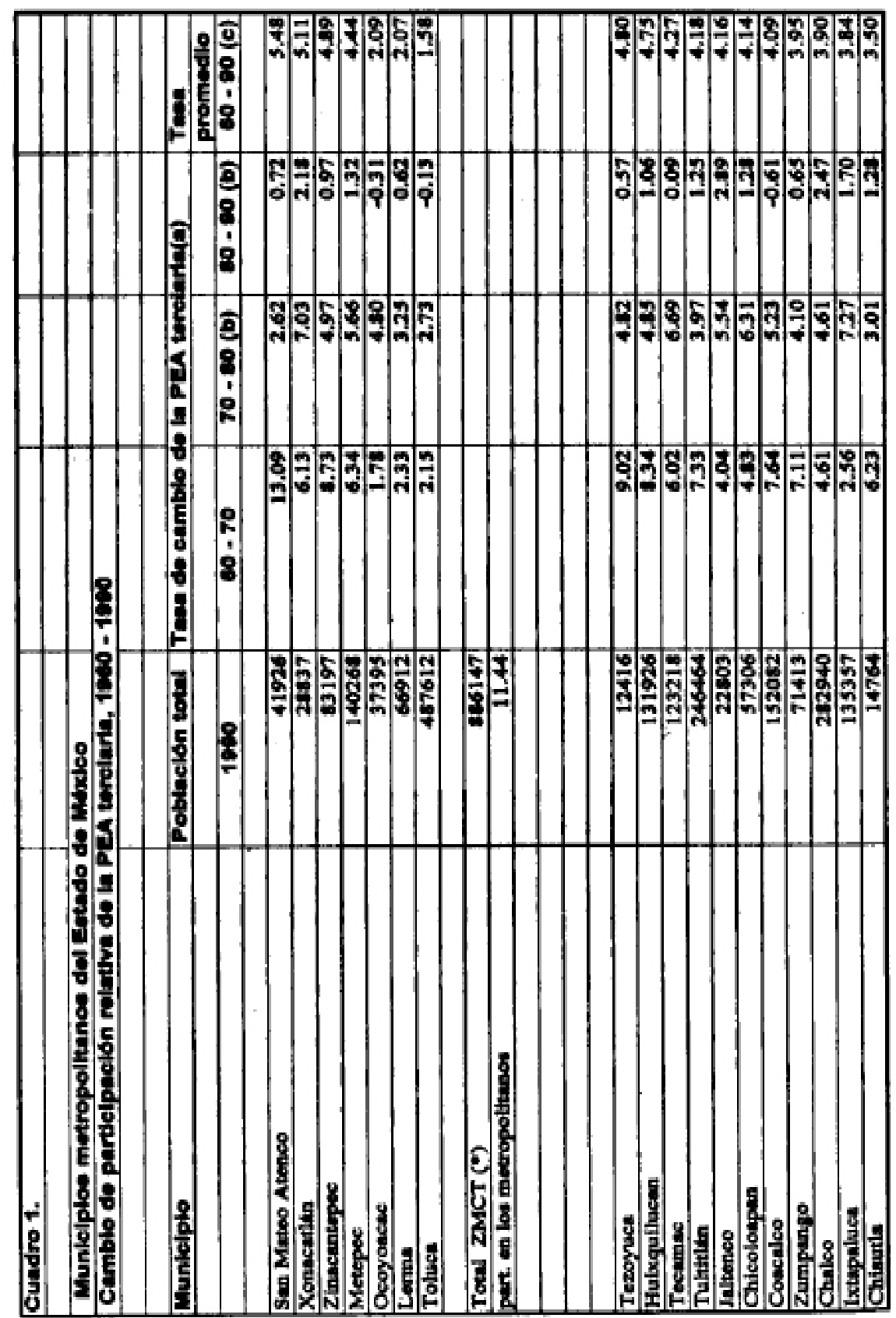




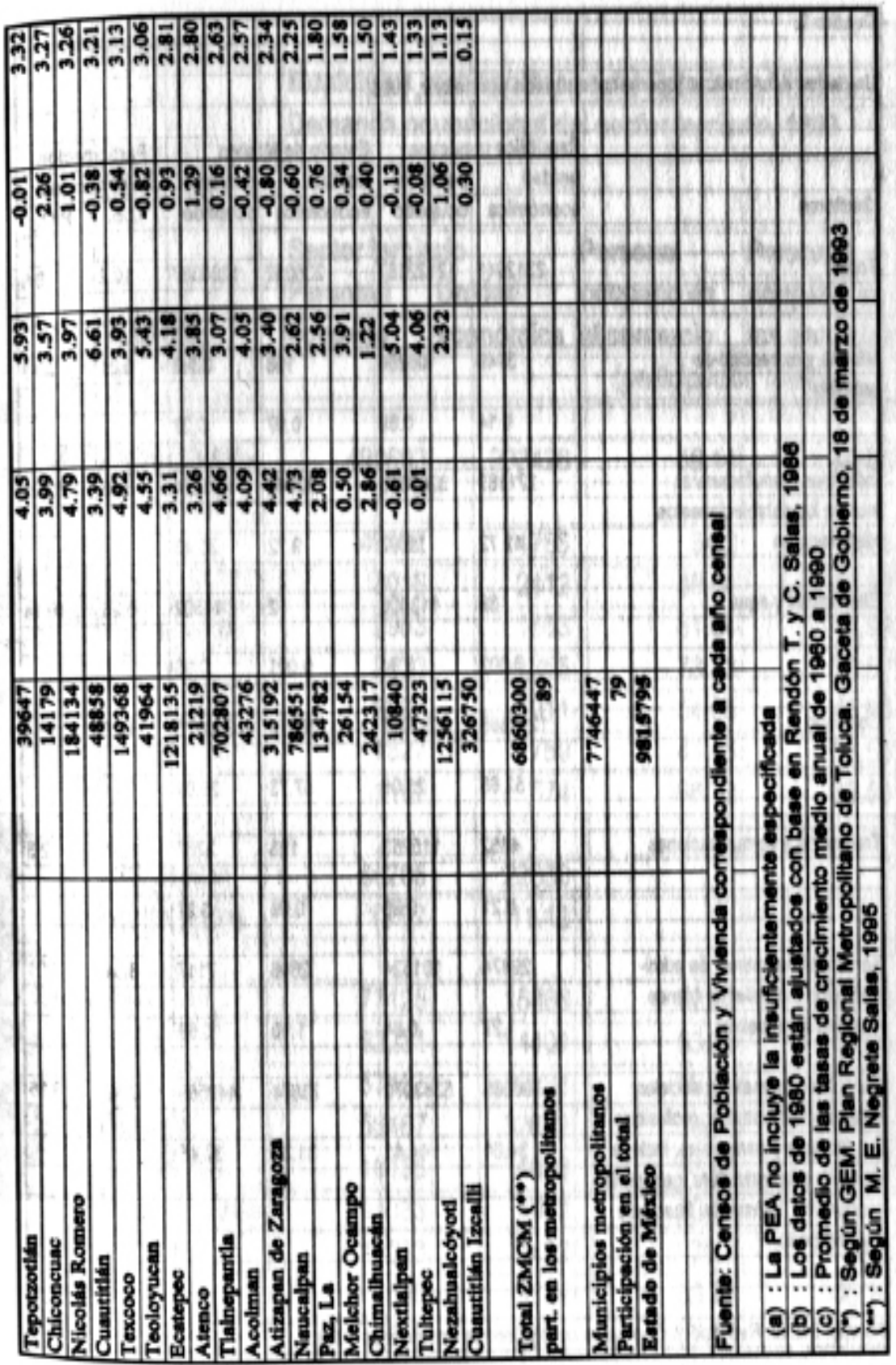


Convergencia Revista de Ciencias Sociales, núm. 12/13, 1996, Universidad Autónoma del Estado de México

\begin{tabular}{|c|c|c|c|c|c|c|}
\hline Cuadro 2. & & & & 1 & & 8 \\
\hline & & & & 8 & T. & \\
\hline Unidades económicas y personal & ocupado por : & sector, 1993 & & & & \\
\hline & & & & & & \\
\hline & República m & vexicana & Estado do IM & exice & Particl & ación \\
\hline 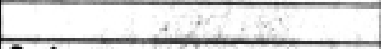 & unidad & personal & unidad & personal & en el na & clonal \\
\hline Sectores & oconómica & ocupado & económica & ocupado & U.E. & P.O. \\
\hline & & & & & & \\
\hline Total & 2312911 & 12122187 & 236052 & 1200387 & 10.2 & 8.9 \\
\hline 32 & +2 & & & & & \\
\hline 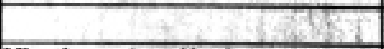 & $7=2$ & & & +9 & 3 & 8 \\
\hline Mineria y extraceclón de & 3149 & 108258 & 158 & 3393 & 5.01 & 3.13 \\
\hline petrolieo & & & & & & \\
\hline & 0.14 & 0.89 & 0.07 & 0.28 & & \\
\hline & & & & & & \\
\hline Industrias manufactureras & 271167 & 3263663 & 22946 & 425274 & 8.46 & 13.03 \\
\hline incluye los establecimientos & & & & & & \\
\hline maquiladores & 11.72 & \begin{tabular}{|r|} 
\\
\end{tabular} & 9.72 & 35.43 & & \\
\hline & & & & & & \\
\hline Electricidad y agua & 38 & 113406 & 2 & 11502 & 5.26 & 10.14 \\
\hline 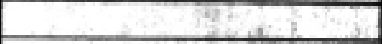 & & & & & & \\
\hline & 0.002 & 0.94 & 0.001 & 0.96 & & \\
\hline 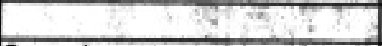 & & & $=x$ & & & \\
\hline $2+x$ & 1217665 & 3037001 & 136273 & 300275 & 11,10 & 9.88 \\
\hline $4 \%+\frac{2 \pi}{2}$ & & & & & & \\
\hline 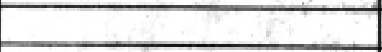 & 52.65 & 25.05 & 57.73 & 25.01 & & \\
\hline & 6 & & & & & \\
\hline Transporte y comunicadiones & 4852 & 115263 & 193 & 3237 & 3.97 & 2.8 \\
\hline 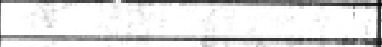 & & & & 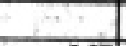 & & \\
\hline & 0.21 & 0.85 & 0.08 & 0.27 & & \\
\hline & & & & & & \\
\hline Servicios financieros, de admi- & 29474 & 101524 & 2606 & 7117 & 8.84 & 7.01 \\
\hline nistración y alquiler de blenes & & & & & & \\
\hline muebles o inmuebles & 1.27 & 0.84 & 1.10 & 0.59 & & \\
\hline & & & & & & \\
\hline Servicios comunales y sociales; & 786566 & 5383070 & 73874 & 449589 & 9.39 & 8.35 \\
\hline hoteles y restaurantes; prolesiona & & & & & & \\
\hline les, tecnicos y personales, incluye & 34.01 & 44.41 & 31.30 & 37.45 & & \\
\hline senvicios a la agricultura, ganaderia, & & & & & & \\
\hline contrucción, transporte, finacieros & & & & & & \\
\hline$y$ al comercio. & E & & & & & \\
\hline 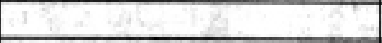 & $z$ & & & & & \\
\hline & & & & & 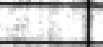 & \\
\hline Fuente: INEGI. Resultados Opoituno & de los Cens & 25 Econdomico & os 1994. & & 5 & \\
\hline
\end{tabular}




\begin{tabular}{|c|c|c|c|c|}
\hline \multirow[t]{2}{*}{ Cuadro 3.} & & & & \\
\hline & \multicolumn{4}{|c|}{ Municipios metropolitanos del Estado de México } \\
\hline 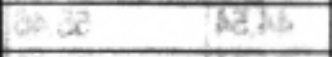 & \multicolumn{4}{|c|}{ Demanda ocupacional del sector terciario, 1993} \\
\hline \multicolumn{5}{|l|}{ 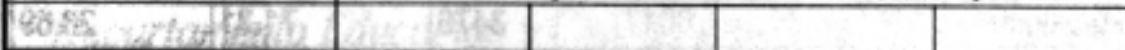 } \\
\hline 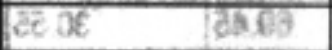 & Dart & & & \\
\hline B.t2 & \multicolumn{2}{|c|}{ Sector terciario } & Personal & Personal \\
\hline A. 23.73 & Personal & Unidad & ocupado en & ocupado en \\
\hline Municipio & ocupado & económica & el comercio & los servicios \\
\hline 0060 & 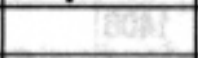 & & \multicolumn{2}{|c|}{ (participación relativa) } \\
\hline 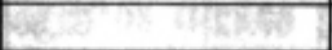 & 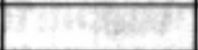 & & & 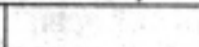 \\
\hline Total Edo. de Méx. & 499693 & 203426 & 59.14 & 40.86 \\
\hline 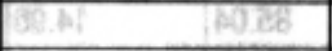 & 20 & 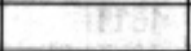 & & \\
\hline Toluca & 47062 & 14766 & 54.2 & 45.8 \\
\hline Metepec & 8016 & 2412 & 49.64 & 50.36 \\
\hline San Mateo Atenco & 2953 & 1653 & 81.04 & 18.96 \\
\hline Zinacantepec & 2323 & 1355 & 73.48 & 26.52 \\
\hline Lerma & 2237 & 1011 & 54.05 & 45.95 \\
\hline Ocoyoacac & 1527 & 759 & 41.45 & 58.55 \\
\hline Xonacatlán $\mathrm{CROES}$ & 1167 & 674 & 66.92 & 33.08 \\
\hline 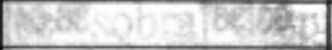 & 20 & 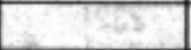 & 8 & 70 \\
\hline Total ZMCT & 65285 & 22630 & & \\
\hline \multirow[t]{2}{*}{ Part. en el total } & 13.07 & 11.12 & $\sqrt{s}$ & 218 \\
\hline & 850 & & & \\
\hline Naucalpan & 77125 & 15866 & 52.22 & 47.78 \\
\hline Tlainepantla & 62231 & 14401 & 53.35 & 46.65 \\
\hline Ecatepec & 57152 & 30231 & 66.59 & 33.41 \\
\hline Nezahualcóyotl & 56467 & 32622 & 58.78 & 41.22 \\
\hline Cuautitlán Izcalli & 18136 & 4656 & 58.49 & 41.51 \\
\hline Atizapan de Zaragoza & 15033 & 4949 & 50.64 & 49.36 \\
\hline Chalco & 14033 & 9422 & 70.8 & 29.2 \\
\hline Texcoco & 9546 & 3724 & 53.76 & 46.24 \\
\hline Chimalhuacán & 8815 & 6581 & 77.18 & 22.82 \\
\hline Paz, La & 8510 & 3698 & 65.76 & 34.24 \\
\hline Coacalco son & 7050 & 2919 & 55.33 & 44.67 \\
\hline Tultitlán & 6912 & 3766 & 63.27 & 36.73 \\
\hline
\end{tabular}


Convergencia Revista de Ciencias Sociales, núm. 12/13, 1996, Universidad Autónoma del Estado de

\begin{tabular}{|l|r|r|r|r|}
\hline Huixquilucan & 6338 & 1729 & 44.54 & 55.46 \\
\hline Ixtapaluca & 6017 & 3476 & 71.31 & 28.69 \\
\hline Nicolás Romero & 5312 & 3143 & 69.45 & 30.55 \\
\hline Tecamac & 4704 & 2613 & 68.84 & 31.16 \\
\hline Cuautitlán & 4306 & 1542 & 61.66 & 38.34 \\
\hline Chiconcuac & 3928 & 2206 & 87.63 & 12.37 \\
\hline Zumpango & 2587 & 1406 & 69.54 & 30.46 \\
\hline Chicoloapan & 2214 & 1496 & 67.12 & 32.88 \\
\hline Tepotzottán 1: & 2117 & 642 & 43.98 & 56.02 \\
\hline Teoloyucan & 1811 & 546 & 85.04 & 14.96 \\
\hline Tultepec & 1335 & 915 & 67.72 & 32.28 \\
\hline Acolman & 1241 & 846 & 71.72 & 28.28 \\
\hline Melchor Ocampo & 839 & 542 & 62.34 & 37.66 \\
\hline Antenco & 594 & 381 & 58.25 & 41.75 \\
\hline Jaltenco & 360 & 292 & 70.83 & 29.17 \\
\hline Tezoyuca & 341 & 215 & 66.86 & 33.14 \\
\hline Nextlalpan & 266 & 155 & 55.26 & 44.74 \\
\hline Chiautla & 232 & 128 & 63.36 & 36.64 \\
\hline & & & & \\
\hline Total ZMCIM & 385552 & 155108 & & \\
\hline Part. en el total & 77 & 76.25 & & \\
\hline & & & & \\
\hline Total municiplos & & & & \\
\hline metropolitanos & 450835 & 177738 & & \\
\hline Part. en el total & 90.22 & 87.37 & & \\
\hline & & & & \\
\hline & & & & \\
\hline Fuente: INEGI. Resultados Oportunos de los Censos Económicos & & & \\
\hline
\end{tabular}

